\title{
Affinage et qualité du Gruyère de Comté \\ III. Fermentation lactique et teneur en acides gras volatils des fromages de Comté
}

\author{
J.L. BERDAGUE *, R. JEUNET et R. GRAPPIN \\ avec la collaboration technique de G. DUBOZ \\ I.N.R.A., Station expérimentale laitière, 39800 Poligny, France \\ * Institut Technique du Gruyère
}

\section{Résumé}

Cet article décrit l'évolution des teneurs moyennes en lactates et en acides gras volatils de C2 à C6 dans le fromage de Comté durant l'affinage.

La composition moyenne du fromage en fin d'affinage (exprimée en mg/100 g de fromage) est la suivante: Lactate $\mathrm{L}(+): 383$; Lactate $\mathrm{D}(-): 295$; Acide acétique (C2): 200 ; Acide propionique (C3) : 222; Acide butyrique (C4) : 17 ; Acide isovalérique (iC5) : 3 ; Acide caproïque (C6) : 9.

L'étude des relations existantes entre les différentes formes optiques des lactates et les acides gras volatils est abordée à plusieurs stades de l'affinage ( 3 semaines, 1 mois et demi, et en fin d'affinage). Le coefficient de corrélation entre lactate L et D passe de $-0,89$ avant affinage à $+0,80$ à la coupe; la corrélation entre $\mathrm{C} 2$ et $\mathrm{C} 3$ augmente régulièrement au cours de l'affinage. En fin d'affinage, il est démontré l'existence de trois groupes de composés indépendants en relation avec la fermentation propionique (Lactates, $\mathrm{C} 2$ et $\mathrm{C} 3$ ), la lipolyse (C4 et $\mathrm{C} 6$ ) et le catabolisme de certaines fractions azotées (iC5). Une évaluation stoechiométrique des composés impliqués dans la fermentation propionique en fin d'affinage montre que les quantités de $\mathrm{C} 2$ et $\mathrm{C} 3$ produites au cours de la phase d'affinage en cave chaude sont liées à la diminution de la teneur en lactates. On observe cependant une quantité de $104 \mathrm{mg} / 100 \mathrm{~g}$ de fromage d'acide acétique non expliqués par la fermentation propionique.

D'après le schéma expérimental mis en œuvre, il apparaît que les conditions d'affinage influencent plus particulièrement la fermentation propionique, les teneurs en C4 et C6 sont modifiées par la saison et la teneur en iC5 dépend essentiellement de la fromagerie.

Mots clés: Fromage - Gruyère de Comté - Affinage - Variations - Lactates - Acides gras volatils.

Ce programme a reçu le soutien financier de la Direction des Industries Agricoles et Alimentaires du Ministère de l'Agriculture (Contrat DIAA $\mathrm{n}^{\circ}$ 81/87) et de l'Association Nationale pour la Recherche et la Technologie (Bourse CIFRE de J.L. Berdagué, convention $n^{\circ}$ 92/82). 


\title{
Summary
}

\author{
Ripening and quality of Gruyère of Comté cheese \\ III. Influence of the ripening conditions on lactic acid and fermentation \\ and on the amount of volatile fatty acids
}

This paper describes the variations of the average amounts of lactate and volatile fatty acids ( $\mathrm{C} 2$ to $\mathrm{C} 6$ ) which occur during cheese maturation.

The mean values (expressed in $\mathrm{mg} / 100 \mathrm{~g}$ of cheese) of mature cheese are : $\mathrm{L}$ Lactate : 383 ; D Lactate : 295 ; acetic acid (C2) : 200 ; propionic acid (C3) : 222 ; butyric acid (C4) : 17 ; isovaleric acid (iC5) : 3 ; caproïc acid (C6) : 9.

Relationship between the various variables were studied at different stages of ripening ( $3 \mathrm{wk}, 1 \mathrm{mo}$. and end of ripening). The correlation coefficient between $\mathrm{L}$ and D lactate changes from $-0,89$ at 3 weeks to $+0,80$ at the end of ripening; the correlation between $\mathrm{C} 2$ and $\mathrm{C} 3$ increases regularly throughout ripening. Using a principal component analysis, it was shown that the quantities of compounds resulting from propionic acid fermentations (lactate, $\mathrm{C} 2$ and $\mathrm{C} 3$ ), lipolysis (C4 and $\mathrm{C} 6$ ) and amino acid degradation (iC5) are independant.

The increase of $\mathrm{C} 2$ and $\mathrm{C} 3$ concentration in cheese during ripening in warm room is closely related to the decrease in the amount of lactate, however a quantity of 104 $\mathrm{mg} / 100 \mathrm{~g}$ of cheese of $\mathrm{C} 2$ is not explained by the propionic acid and fermentation.

The ripening conditions influence mainly the propionic acid fermentation, while $\mathrm{C} 4$ and $\mathrm{C} 6$ contents are under the influence of the season, and the amount of iC5 is closely related to the origin of the cheese.

Key words: Cheese - Gruyère of Comté - Ripening - Variations - Lactates - Volatile fatty acids.

\section{Introduction}

Le dosage par chromatographie en phase gazeuse des acides gras volatils de C2 à C6 du fromage (Kuzdzal-Savoie et Kuzdzal, $1966 ; 1967 ; 1971$; 1974) ou de $\mathrm{C}_{2}$ à $\mathrm{C}_{4}$ (FAVRE-Bonvin, 1984) est actuellement largement employé par l'industrie fromagère pour caractériser soit la fermentation butyrique, soit les anomalies de la fermentation propionique ou de la lipolyse des triglycérides au cours de l'affinage des fromages à pâte cuite pressée. (KuzDZAL-SAvoie et Kuzdzal, 1966 ; Institut Technique du Gruyère, 1980 ; MAYenoBe et al., 1983 ; Снамва, 1984). Le dosage des différentes formes de lactates : L (+) et $\mathrm{D}(-)$, est également un moyen précis permettant de mesurer à un stade précoce l'activité des ferments lactiques thermophiles.

Cette étude, qui s'inscrit dans le cadre du programme de travail décrit par GrapPIN et al. (1987), sur l'affinage et la qualité du Comté, a pour but de mesurer l'évolution des teneurs en lactates et en acides gras volatils au cours de l'affinage, et de déterminer l'influence des conditions d'affinage des fromages, en fonction de la fromagerie (qui regroupe la qualité du lait et la technique de fabrication) et de la saison de fabrication. 


\section{Matériel et méthode}

\section{A. Protocole expérimental}

Ce dernier a été décrit en détail par GrapPin et al. (1987). Rappelons que les 96 fromages de l'étude représentent 24 fabrications réalisées dans 4 fromageries $(\mathrm{F} 1, \mathrm{~F} 2, \mathrm{~F} 3$ et $\mathrm{F} 4)$ sur une période de 6 mois $(3$ mois d'hiver et 3 mois d'été). Au cours de chaque fabrication, 4 fromages ont été obtenus à partir d'une même cuve. Ces 4 fromages, considérés comme étant de composition identique, ont été affinés par 4 affineurs différents (A1, A2, A3 et A4). Le schéma expérimental correspond à un plan factoriel dont les niveaux sont répartis comme suit : Fromagerie (4 niveaux), Affinage (4 niveaux), Saison de fabrication (2 niveaux), Mois (3 répétitions).

\section{B. Analyses chimiques}

\section{Echantillons}

- Stade de prélèvement des échantillons : chacun des 96 fromages étudiés a été analysé à différents stades de l'affinage. Le galactose et les lactates ont été dosés sur les fromages à $20 \mathrm{~h}$ (stade F). Les lactates et les acides gras volatils ont été dosés à 3 semaines (stade A), 9 semaines (stade P) et en fin d'affinage entre 4 et 9 mois (stade C).

Technique d'échantillonnage : les échantillons, prélevés en milieu de rayon à l'aide d'une sonde de diamètre interne $20 \mathrm{~mm}$, ont été aussitôt emballés dans du papier aluminium sulfurisé, refroidis à $4{ }^{\circ} \mathrm{C}$ et congelés à $-20^{\circ} \mathrm{C}$ jusqu'au moment de l'analyse. La prise d'essai est de $10 \mathrm{~g}$ de fromage dans le cas du dosage du galactose et des lactates et de $20 \mathrm{~g}$ pour l'extraction et le dosage des acides gras volatils.

\section{Méthodes}

- Galactose et lactates : les dosages sont effectués sur les filtrats aqueux obtenus selon la méthode de Kuchroo et Fox (1982).

Le galactose (GAL : mg/100 g de fromage) est dosé par méthode enzymatique (Boehringer-ManNheim, réf. 176-303) selon la technique de Kurz et WALLENFELS (1974).

Les lactates $\mathrm{L}(+)$ et $\mathrm{D}(-)$ (LAL et LAD : $\mathrm{mg} / 100 \mathrm{~g}$ de fromage) sont dosés par méthode enzymatique (BoEHRINGER-MANNHEIM, réf. 139-084).

- Les acides gras volatils (AGV : $\mathrm{mg} / 100 \mathrm{~g}$ de fromage) sont dosés par chromatographie en phase gazeuse après extraction selon une technique mise au point par Kuzdzal-Savole (1966) et adaptée par AGNeT (1979). Il s'agit d'une extraction des graisses et des acides gras libres par épuisement sous flux d'un mélange $50-50(\mathrm{~V} / \mathrm{V})$ de diéthyl-oxyde et éther de pétrole $\left(35-60^{\circ} \mathrm{C}\right)$. Les acides gras libres sont séparés des graisses par émulsion et décantation entre la phase éther et une phase hydro-éthanolique, après neutralisation par de la soude diluée. Les acides gras sont stockés sous forme de savon avant chroma- 
tographie. Lors de la chromatographie, ceux-ci sont libérés de leur combinaison saline par acidification à l'acide trichloracétique. Les conditions chromatographiques sont décrites par Berdague (1986).

Les acides gras volatils qu'il est possible de suivre par cette technique sont : l'acide acétique (C2), l'acide propionique (C3), l'acide isobutyrique (iC4), l'acide butyrique (C4), l'acide isovalérique (iC5), l'acide valérique = étalon interne (C5), l'acide isocaproïque (iC6) et l'acide caproïque (C6).

\section{Résultats et discussion}

\section{A. Evolution de la composition moyenne des fromages en cours d'affinage}

Parmi les composants analysés, les acides isobutyriques et isocaproïque ne sont détectés qu'épisodiquement et en très faible quantité (inférieure à $1 \mathrm{mg}$ par $100 \mathrm{~g}$ de fromage). Il en est de même pour le galactose, produit d'hydrolyse du lactose, qui n'apparaît qu'exceptionnellement 20 heures après fabrication et uniquement dans la seule fromagerie $\mathrm{F} 4$, à raison de 36 à 450 $\mathrm{mg} / 100 \mathrm{~g}$ de fromage. Au-delà de 20 heures, le galactose est totalement dégradé et ne sera plus mis en évidence.

L'évolution moyenne des autres composants est représentée sur la figure 1. On constate que le LAL est à sa concentration maximum à 20 heures à raison de $963 \mathrm{mg} / 100 \mathrm{~g}$ de fromage et décroît d'environ $60 \%$ durant l'affinage.

Le LAD est dosé en plus faible concentration, soit $306 \mathrm{mg} / 100 \mathrm{~g}$ de fromage à 20 heures. Sa teneur maximale se situe à $460 \mathrm{mg} / 100 \mathrm{~g}$ de fromage après un mois et demi d'affinage. Rappelons que la configuration stérique des lactates est très influencée par la nature des levains. Durant la fermentation lactique, $S$. thermophilus se développe le premier après deux heures de pressage et ne produit que LAL. Par contre, les lactobacilles se multiplient plus tardivement, ne produisant de l'acide lactique qu'après quatre heures de pressage (AcColas et Veaux, 1978). L. helveticus produit la même quantité de LAL et de LAD, tandis que $L$. lactis produit uniquement LAD (STEFFEN et BLANC, 1971). Les mêmes auteurs signalent aussi l'influence des conditions de culture (phase de croissance et milieu nutritionnel) sur les proportions des deux isomères pour une même espèce bactérienne. Les teneurs en lactates observées dans cette étude (fig. 1) sont proches de celles rencontrées dans la littérature (Mocquot, 1978 ; TuRner et Thomas, 1980 ; Deiana et al., 1984). Les teneurs en AGV sont également des valeurs couramment rencontrées dans le cas de Comtés non butyriques (Kuzdzal-Savole et Kuzdzal, 1967, 1974). Les teneurs élevées en acide propionique, soit en moyenne $200 \mathrm{mg} / 100 \mathrm{~g}$ de fromage en fin d'affinage, sont caractéristiques des fromages à pâtte pressée cuite. Les teneurs en acide caproïque du Comté, de l'ordre de $9 \mathrm{mg} / 100 \mathrm{~g}$ de fromage, apparaissent légèrement plus élevées que les teneurs observées en Emmental (2 à $8 \mathrm{mg} / 100 \mathrm{~g}$ de fromage, ITG 1985). L'affinage plus long des fromages de Comté peut expliquer cette différence. La teneur en acide isovalérique, produit de dégradation de la leucine (BARAud et al., 1970) est de l'ordre de $3 \mathrm{mg} / 100 \mathrm{~g}$ de fromage. 


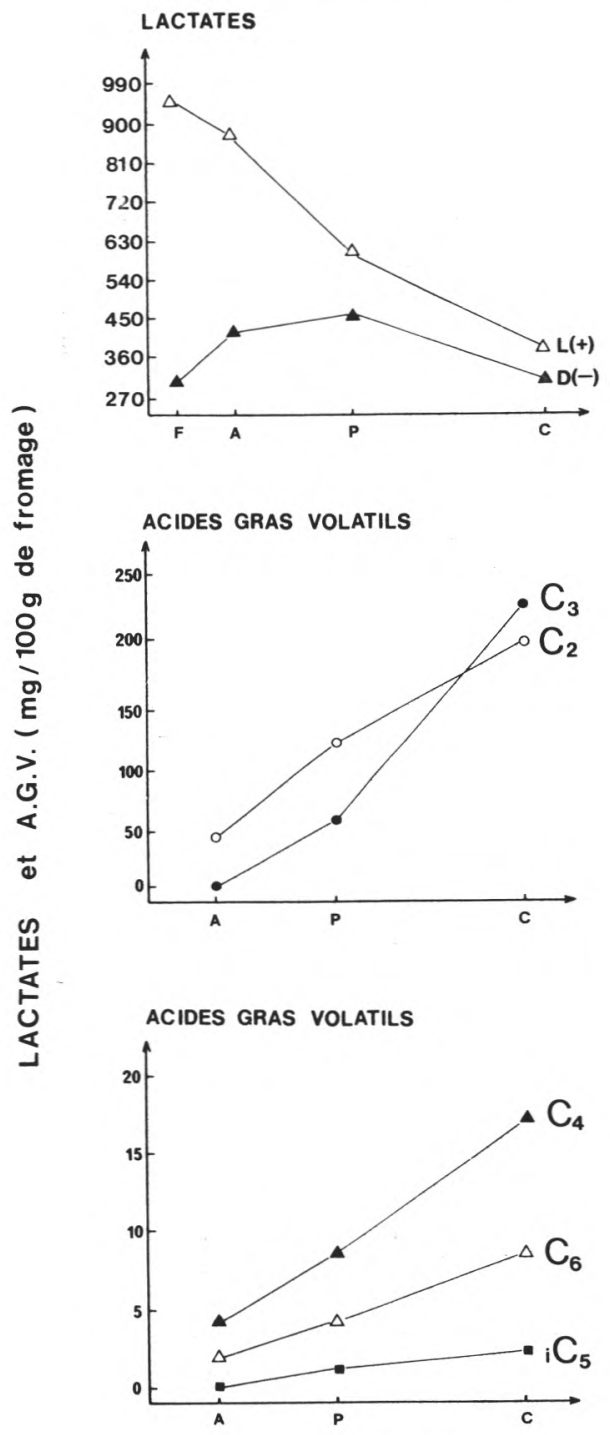

STADE D AFFINAGE

Fig. 1

Evolution au cours de l'affinage de la teneur moyenne en lactates et acides gras volatils de C2 à C6 dans le fromage de Comté.

$F: 20 h ; A: 3$ semaines; $P$ : 6 semaines ; $C$ : Coupe.

Variation during ripening of the average amount of lactates and volatile fatty acids (C2 to C6) in Comté cheese.

$F: 20 h ; A: 3$ weeks ; $P: 6$ weeks ; $C$ : End of ripening. 
TABLEAU I

Evolution en cours d'affinage ( $A: 3$ semaines ; $P: 6$ semaines; $C:$ à la coupe des fromages) des coefficients de corrélation entre l'acide acétique : C2, l'acide propionique : C3 et les lactates $L(+): L A L$ et D (-) : LAD (Seuils de signification :

$$
p=0,01 ; r=0,33)
$$

Matrix of the coefficients of correlation between lactate $L(L A L)$, lactate $D(L A D)$, acetic acid (C2) and propionic acid (C3) during Comté cheese maturation: 3 weeks $(A), 6$ weeks $(P)$ and end of the ripening period $(C)$ (level of signification: $p=0.01 ; r=0.33$ )

\begin{tabular}{c|r|r|r|r|r|r|r|r|r|r|r|r}
\hline & \multicolumn{4}{|c|}{ C2 } & \multicolumn{3}{c|}{ C3 } & \multicolumn{3}{c}{ LAL } & \multicolumn{1}{c}{ LAD } \\
\hline Stade & A & \multicolumn{1}{|c|}{ P } & C & A & P & C & A & P & C & A & P & C \\
\hline C2 & 1.00 & 1.00 & 1.00 & & & & & & & & & \\
\hline C3 & 0.33 & 0.41 & 0.80 & 1.00 & 1.00 & 1.00 & & & & & & \\
\hline LAL & -0.99 & -0.14 & -0.70 & -0.07 & -0.67 & -0.90 & 1.00 & 1.00 & 1.00 & & & \\
\hline LAD & 0.00 & -0.48 & -0.68 & 0.00 & -0.59 & -0.85 & -0.89 & -0.21 & +0.90 & 1.00 & 1.00 & 1.00 \\
\hline
\end{tabular}


La comparaison des taux d'acides acétique et propionique (fig. 1) montre des teneurs déjà élevées d'acide acétique en début d'affinage aux stades A (36 $\mathrm{mg} / 100 \mathrm{~g}$ de fromage) et $\mathrm{P}$ (113 $\mathrm{mg} / 100 \mathrm{~g}$ de fromage), tandis que l'acide propionique n'est présent qu'à l'état de traces au stade $\mathrm{A}(2 \mathrm{mg} / 100 \mathrm{~g}$ de fromage) et en plus faible quantité que l'acide acétique au stade P (52 mg/100 $\mathrm{g}$ de fromage).

\section{B. Relations entre acides gras volatils et lactates}

\section{Evolution au cours de l'affinage des relations entre variables liées à la} fermentation propionique $(\mathrm{C} 2, \mathrm{C} 3, \mathrm{LAL}$ et LAD)

Les corrélations à divers stades de l'affinage du fromage entre lactates, acétate et propionate, donnent une vue dynamique des différents phénomènes liés à la fermentation propionique (tabl. 1).

$\mathrm{Au}$ cours des différents stades, les coefficients de corrélation entre les variables augmentent systématiquement pour devenir hautement significatifs, excepté dans le cas de la relation entre LAL et LAD qui s'inverse au cours du temps (le coefficient de corrélation passe de $-0,89$ à 0,21 pour atteindre 0,90 au stade coupe). Cette inversion de la corrélation entre les deux formes optiques des lactates est une illustration des mécanismes de racémisation intervenant au cours d'affinage. Le rapport entre les formes de lactate en fin d'affinage est proche de 1 , ainsi qu'en témoigne la pente de l'équation de régression $\mathrm{LAL}=1,15 \mathrm{LAD}+5,02$. A ce stade, la mesure séparée des isomères optiques des lactates n'apporte pas plus d'information que celle des lactates totaux. Au fur et à mesure que se poursuit l'affinage, l'acide acétique apparaît de plus en plus corrélé à l'acide propionique. Le coefficient de corrélation évolue de 0,33 à 0,41 puis 0,80 . La corrélation négative entre $\mathrm{C} 3$ et les lactates ne devient significative qu'à partir du moment où la fermentation propionique a commencé, c'est-à-dire au stade $\mathrm{P}$. Cette corrélation est meilleure avec LAL $(\mathrm{r}=-0,67)$ qu'avec LAD $(\mathrm{r}=-0,59)$. A l'inverse, on observe que le $\mathrm{C} 2$ est mieux corrélé au stade P avec LAD $(r=-0,48)$ qu'avec LAL $(r=-0,14$; Non Significatif).

Les très fortes corrélations négatives entre l'acide acétique et LAL ou LAD ( $\mathrm{r}=-0,70$ et $-0,68)$ ou entre l'acide acétique et l'acide propionique ou les lactates $(\mathrm{r}=-0,90$ et $-0,85)$ ne s'observent que sur des fromages affinés.

Les lactates totaux consommés sont très bien corrélés à l'acétate : $r=0,73$ et au propionate : $r=0,90$.

\section{Relations entre acides gras volatils et lactates en fin d'affinage}

L'analyse en composantes principales des acides gras volatils et des lactates donne une synthèse des relations qui existent entre ces variables (fig. 2). On obtient ainsi trois groupes indépendants de variables ayant des corrélations élevées (supérieures à 0,84 ) avec les trois premiers axes :

- lactates, acétate et propionate, qui caractérisent la fermentation propionique, apparaissent très fortement corrélés à l'axe 1 ; 


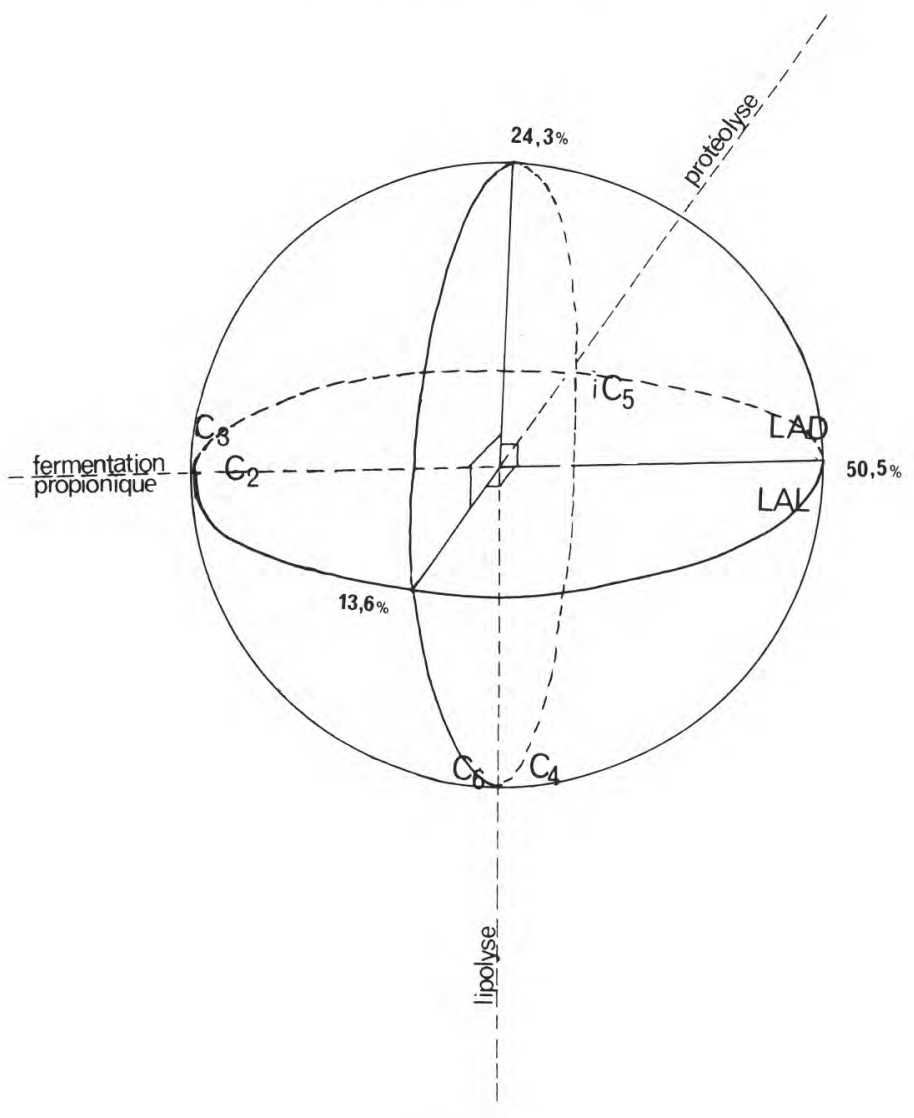

Fig. 2

Sphère des corrélations au stade coupe dans l'analyse en composantes principales des 7 variables (acides gras volatils et lactates).

Sphere of correlations in principal components analysis of the amount of $L$ and $D$ lactate ( $L A L, L A D)$ and volatile fatty acids $(C 2, C 3, C 4, C 6, i C 5)$ of mature Comté cheese.

- les acides butyrique et caproïque sont très fortement corrélés à l'axe 2 qui caractérise la lipolyse ; axe.

- l'acide isovalérique qui est un indice de protéolyse définit le troisième

3. Evaluation stoechiométrique du bilan de la fermentation propionique en Comté

Pour le calcul des régressions, les quantités d'AGV et de lactates ne sont plus exprimées en $\mathrm{mg} / 100 \mathrm{~g}$ mais en $\mu \mathrm{Mole} / \mathrm{g}$ de fromage. L'acide lactique total consommé est estimé pour les 96 fromages en calculant la différence entre les quantités de lactates présentes à trois semaines (avant début de la 
fermentation propionique en cave chaude) et celles restantes au stade coupe. Dans ces conditions, il est possible de confronter les résultats expérimentaux à ceux que prévoit la théorie.

D'après l'équation de Fitz (CLARK, 1912), une mole de lactates consommée au cours de la fermentation propionique donne 0,33 mole de $\mathrm{C} 2$ et 0,66 mole de C3. Les équations de régression calculées :

$\mathrm{C} 2=0,26$ lactates $+15,45(\mathrm{r}=0,72)$ et $\mathrm{C} 3=0,48$ lactates $-3,04(\mathrm{r}=0,90)$ montrent des coefficients de régression assez proches des valeurs théoriques, soit 0,26 contre 0,33 et 0,48 contre 0,66 , et un rapport $\mathrm{C} 2 / \mathrm{C} 3$ de $0,26 / 0,48$, égal à 1,8 et voisin du rapport 2 prévu dans l'équation de FITz. VAN NIEL (1928) a trouvé des valeurs comprises entre 1,6 et 1,8 et d'après WooD et WERKMANN (1937), ce rapport peut varier énormément entre 2,1 et 14,7. Sur le plan pondéral, le rapport $\mathrm{C} 3 / \mathrm{C} 2$ pour les 96 fromages est de 1,12 contre 22,3 dans les fromages à pâte pressée cuite (Kuzdzal-Savoie, 1974).

Lorsque l'on calcule la régression $\mathrm{C} 2=0,53 \mathrm{C} 3+17,80$, on constate l'existence d'une quantité significative de 17,30 Mole/g (ou $104 \mathrm{mg} / 100 \mathrm{~g}$ de fromage) de C2 dont l'origine n'est pas expliquée par la fermentation propionique caractérisée par l'acide propionique. Une déviation précoce du métabolisme des glucides, non conforme à l'équation de FrTz, est envisageable pour expliquer cette formation de C2. Il a déjà été mentionné que les bactéries lactiques étaient capables de produire du C2, depuis les sucres, à partir du pyruvate, sans passer par les lactates (CHOIsy, 1984). Les conditions moins réductrices des premiers jours des fromages seraient propices à cette production. Il est possible également que d'autres microorganismes soient capables d'utiliser les sucres pour former du C2. Une part de C2 peut aussi provenir du catabolisme des matières azotées solubles (peptides, acides aminés).

\section{Relations entre acide butyrique et acide caprö̈que}

Pour l'ensemble des fromages, le rapport pondéral C4/C6 est voisin de 2. Selon Kuzdzal-Savoie (1974), un tel rapport (proche de la proportion de ces acides dans les glycérides du fromage) correspond à une lipolyse des triglycérides.

\section{Facteurs de variation des quantités d'acide lactique et d'acides} gras volatils dans les fromages en fin d'affinage

Le tableau 2 regroupe, en fonction des facteurs étudiés, les valeurs moyennes obtenues pour chacune des sept variables. L'influence des trois facteurs (affinage, fromagerie et saison) a été étudiée par analyse de la variance dont les principaux résultats sont regroupés dans le tableau 3. Selon les variables, les facteurs affinage, fromagerie et saison de fabrication permettent d'expliquer de 23,7 à $66,1 \%$ des variations. On constate que les composés liés à la fermentation propionique, à la lipolyse ou à la protéolyse ne sont pas influencés de la même manière par les différents facteurs.

\section{Teneurs en lactates, acétate et propionate}

On note une influence hautement significative du facteur affinage sur les teneurs en $\mathrm{C} 2, \mathrm{C} 3$ et lactates des fromages affinés. 


\section{TABLEAU II}

Valeurs moyennes des teneurs en lactates et en acides gras volatils des fromages en fin d'affinage (exprimées en mg/100 $\mathrm{g}$ de fromage), classées en fonction des facteurs affinage, fromagerie et saison

Mean values of $L$ and $D$ Lactate and volatile fatty acids contents of mature cheese (in $\mathrm{mg} / 100 \mathrm{~g}$ of cheese), classified according to the factors : ripening conditions, cheese origin (cheese plant) and season

\begin{tabular}{|c|c|c|c|c|c|c|c|c|c|c|c|}
\hline \multirow{2}{*}{ Variable } & & \multicolumn{4}{|c|}{$\begin{array}{c}\text { Affinage } \\
\text { Ripening conditions }\end{array}$} & \multicolumn{4}{|c|}{$\begin{array}{l}\text { Fromagerie } \\
\text { Cheese plant }\end{array}$} & \multicolumn{2}{|c|}{$\begin{array}{l}\text { Saison } \\
\text { Season }\end{array}$} \\
\hline & & A1 & A2 & A3 & A4 & $\mathrm{F} 1$ & $\mathrm{~F} 2$ & F3 & $\mathrm{F} 4$ & $\begin{array}{l}\text { Hiver } \\
\text { Winter }\end{array}$ & $\begin{array}{c}\text { Eté } \\
\text { Summer }\end{array}$ \\
\hline \multirow[t]{2}{*}{ Acide lactique } & $\mathrm{L}$ & 441 & 267 & 202 & 269 & 265 & 332 & 226 & 356 & 286 & 304 \\
\hline & $\mathrm{D}$ & 574 & 340 & 279 & 341 & 370 & 465 & 275 & 423 & 340 & 427 \\
\hline Lactic acid & Total & 1015 & 607 & 481 & 610 & 635 & 797 & 501 & 779 & 626 & 731 \\
\hline \multirow{2}{*}{ Acides gras volatils } & $\mathrm{C} 3$ & 86 & 254 & 302 & 246 & 211 & 175 & 277 & 225 & 253 & 191 \\
\hline & $\mathrm{C} 4$ & 13 & 19 & 19 & 17 & 21 & 16 & 17 & 14 & 12 & 22 \\
\hline \multirow[t]{2}{*}{ Volatile fatty acids } & C6 & 7 & 9 & 11 & 9 & 11 & 8 & 9 & 8 & 6 & 12 \\
\hline & iC5 & 2 & 3 & 3 & 2 & 2 & 4 & 3 & 1 & 3 & 2 \\
\hline
\end{tabular}




\section{TABLEAU III}

Résultats d'analyses de la variance des teneurs en lactates et acides gras volatils des fromages au stade coupe: pourcentage des variances expliquées

$$
\text { par les différents facteurs }
$$

Results of variance analyses of the lactactes and volatile fatty acids contents of mature cheeses: percent of the variances explained by the different factors

\begin{tabular}{|c|c|c|c|c|c|}
\hline \multirow[b]{2}{*}{$\begin{array}{l}\text { Variable } \\
\text { Variable }\end{array}$} & & \multicolumn{4}{|c|}{ Facteurs (Factors) } \\
\hline & & $\begin{array}{c}\text { Affinage } \\
\text { Ripening } \\
\text { conditions }\end{array}$ & $\begin{array}{l}\text { Fromagerie } \\
\text { Cheese plant }\end{array}$ & $\begin{array}{l}\text { Saison } \\
\text { Season }\end{array}$ & $\begin{array}{l}\text { Total } \\
\text { Total }\end{array}$ \\
\hline \multirow{2}{*}{ Lactates } & $\mathrm{L}$ & $42.8 * *$ & $16.9 * *$ & $6.4^{* *}$ & 66.1 \\
\hline & D & $42.9 * *$ & $14.6^{* *}$ & N.S. & 57.5 \\
\hline \multirow{2}{*}{$\begin{array}{l}\text { Acides gras } \\
\text { volatils }\end{array}$} & $\mathrm{C} 2$ & $26.4 *$ & $8.2 *$ & N.S. & 34.6 \\
\hline & C3 & $42.7^{* *}$ & $8.8^{* *}$ & $6.3^{* *}$ & 57.8 \\
\hline \multirow{3}{*}{$\begin{array}{l}\text { Volatile fatty } \\
\text { acids }\end{array}$} & $\mathrm{C} 4$ & $6.0 *$ & $9.3 * *$ & $30.9 * *$ & 46.2 \\
\hline & C6 & N.S. & N.S. & $23.7^{* *}$ & 23.7 \\
\hline & iC5 & N.S. & $32.2 * *$ & N.S. & 32.2 \\
\hline \multicolumn{6}{|c|}{$\begin{array}{ll}\text { N.S. } & \text { : non significatif } \\
& \text { non significant } \\
& \text { significatif } P<0,05 \\
& \text { significant } P<0.05 \\
& : \text { significatif } P<0,01 \\
& \text { significant } P<0.01\end{array}$} \\
\hline
\end{tabular}

La température des caves joue ici un rôle prépondérant (fig. 3a), en augmentant l'activité des bactéries propioniques. L'affinage basse température donne des taux moyens de $\mathrm{C} 2$ et $\mathrm{C} 3$ respectifs de 140 et $87 \mathrm{mg} / 100 \mathrm{~g}$ de fromage contre 220 à $267 \mathrm{mg} / 100 \mathrm{~g}$ de fromage dans le cas d'affinage à 16$18^{\circ} \mathrm{C}$. L'influence de la fromagerie vient en second plan et peut s'expliquer par des flores naturelles et des ensemencements variés des laits. L'influence saisonnière peut trouver une explication du même type.

\section{Teneurs en acides butyrique et caprö̈que}

- Le facteur saison de fabrication explique la quasi-totalité (30,9 et $23,7 \%$ ) des variations significatives de la teneur en C4 et C6 (tabl. 2).

Durant la période estivale, les taux de $\mathrm{C} 4$ et $\mathrm{C} 6$ sont beaucoup plus élevés que durant la période hivernale. Cet effet saison est très visible selon l'axe 2 de la figure $3 \mathrm{~b}$, où l'on note une nette séparation dans le plan 1-2 des deux populations de fromages "été et hiver ». La teneur moyenne en C4 et C6 est respectivement de 12,0 et $5,8 \mathrm{mg} / 100 \mathrm{~g}$ de fromage en hiver et de 21,6 et 11,6 $\mathrm{mg} / 100 \mathrm{~g}$ de fromage en été (tabl. 2). 

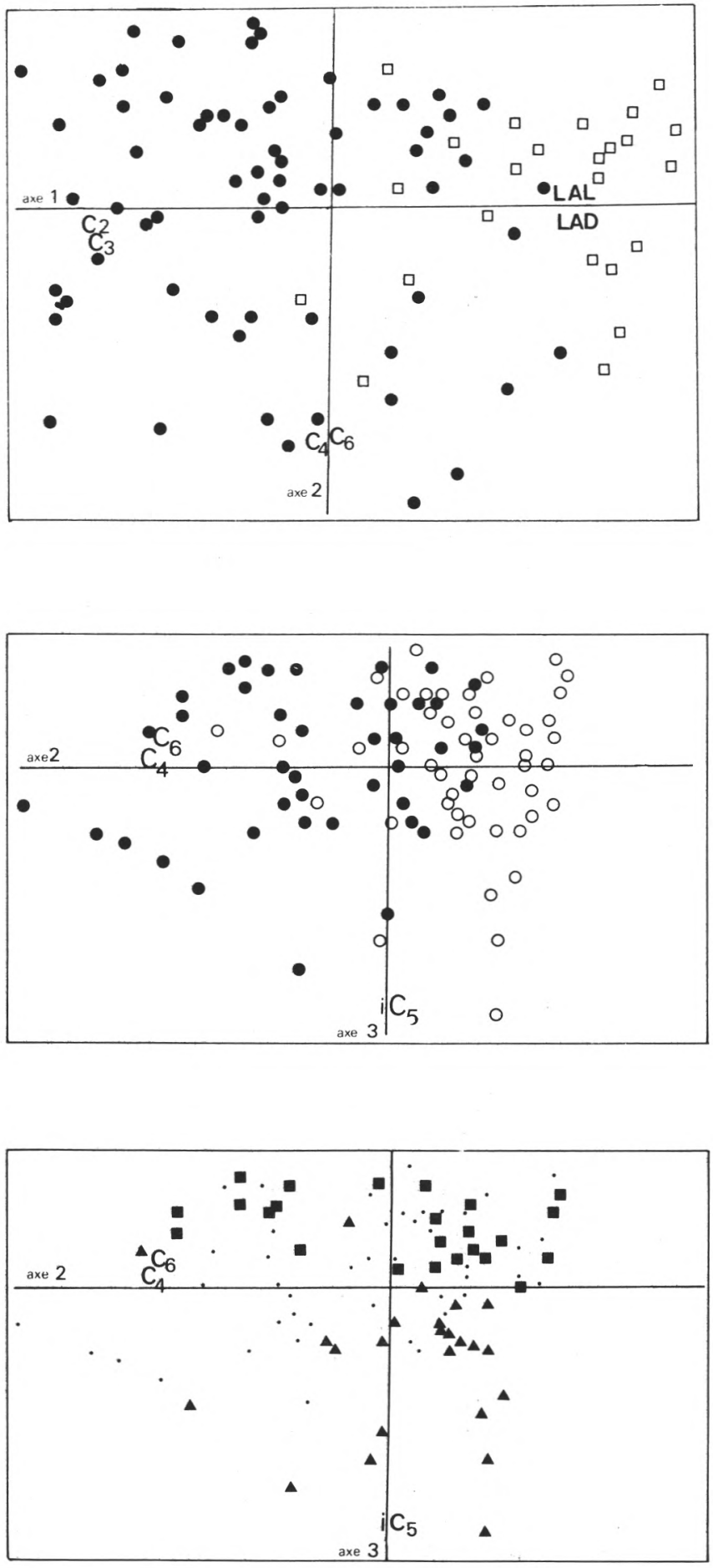
Soulignons que les teneurs en $\mathrm{C} 4$ et $\mathrm{C} 6$ des fromages ne varient pas comme celles des triglycérides du lait qui sont, à l'inverse, plus riches en $\mathrm{C} 4$ et C6 en hiver et fin de printemps qu'en été (MAsson et al., 1978). Dans les fromages, les teneurs les plus faibles observées correspondent aux mois de février, mars et avril, les plus fortes aux mois d'août, septembre et octobre. Les variations antagonistes des $\mathrm{C} 4$ et $\mathrm{C} 6$ libres dans les fromages, et des $\mathrm{C} 4$ et C6 liés au glycérol dans les laits, peuvent signifier que la principale cause des variations saisonnières, observées dans le fromage, n'est pas la conséquence directe du changement de mode d'alimentation des vaches mais plutôt celle de variations des potentiels lipolytiques dans les fromages dont l'origine peut être à rechercher au niveau du lait.

- Un effet fromagerie, hautement significatif mais de moindre amplitude que l'effet saison de fabrication est observé, la fromagerie F1 présentant les teneurs les plus élevées (tabl. 2). Ce facteur n'explique que 9,3\% des variations du taux de $\mathrm{C} 4$ en fin d'affinage.

- L'effet du facteur affinage sur les teneurs en acide butyrique et caproïque est faible (C4) ou non significatif (C6).

\section{Teneur en acide isovalérique}

Le taux d'acide isovalérique des fromages est particulièrement influencé par la fromagerie qui explique $32 \%$ des variations au stade coupe, dans les fromages. La figure $3 \mathrm{c}$ montre nettement que deux fromageries $\dot{\mathrm{F}} 2$ et $\mathrm{F} 4$ s'opposent pour leur teneur en acide isovalérique. L'origine de cette influence significative de la fromagerie est liée à des potentiels protéolytiques différents des laits et des levains de chaque fromagerie. Les facteurs affinage et saison n'influent pas significativement sur le taux d'iC5 au stade coupe.

Fig. 3

Analyse en composantes principales des teneurs en lactates et acides gras volatils des fromages au stade coupe.

Principal components analyses of the amount of $L$ and $D$ lactates ( $L A L, L A D)$ and volatile fatty acids $(\mathrm{C} 2, \mathrm{C3}, \mathrm{C} 4, \mathrm{C} 6, i \mathrm{C5})$ in Comté cheese at the end of the ripening period.

$3 a$ : influence de la température d'affinage sur les teneurs en C2, C3 et lactates.

influence of the ripening temperature on the amount of $L A L, L A D$ and $C 2, C 3$.

$\square$ : $A 1\left(10-12{ }^{\circ} \mathrm{C}\right)$

- : $A 2, A 3, A 4,\left(16-18^{\circ} \mathrm{C}\right)$

$3 b$ : Influence de la saison sur les teneurs en C4 et C6

Influence of the season on the amount of $C 4$ and $C 6$

0 : hiver; winter

- : été ; summer

$3 c$ : Influence de la fromagerie sur la teneur en iC5

Influence of the cheese origin (cheese plant) on the amount of iC5

두 : F4

$\Delta: F 2$

- :F1 et $F 3$ 


\section{Conclusion}

Cette étude montre que dans le cas de fromages de Comté non butyriques, l'analyse simultanée des lactates et des acides gras volatils met en évidence une indépendance quasi-totale entre les mécanismes de la fermentation propionique, de la lipolyse des glycérides et du catabolisme de la leucine, ce qui confirme l'intérêt du dosage des A.G.V. comme profil de l'évolution biochimique des principaux constituants des fromages.

Le dosage des A.G.V. et des lactates montre qu'une quantité d'acide acétique $(104 \mathrm{mg} / 100 \mathrm{~g}$ de fromage en moyenne) apparaît précocement dans les fromages, indépendamment de la fermentation propionique. En revanche, les relations entre les lactates consommés en cave chaude et la production correspondante de $\mathrm{C} 2$ et $\mathrm{C} 3$ sont très proches de celle de l'équation théorique de FITz.

La fermentation propionique est essentiellement influencée par la température d'affinage. Quant à la lipolyse, estimée à partir des acides butyrique et caproïque libres, elle varie essentiellement selon la saison de fabrication des fromages, mais aussi en partie avec l'origine des fromages et correspond soit à une flore psychrotrophe élevée (été) soit à des fromageries effectuant un report de lait pendant 24 heures (fromagerie F1). La dégradation de la leucine apparaît très liée à des potentiels propres à chaque fromagerie.

Reçu le 9 juillet 1986.

Accepté pour publication le 7 novembre 1986.

\section{Références bibliographiques}

Accolas J.P., Veaux M., 1978. Evolution de la flore lactique thermophile au cours du pressage des fromages à pâte cuite. Lait, 58, 118-132.

Agnet Y., 1979. Note technique non publiée sur l'analyse des acides gras volatils de C2 à C6. Laboratoire Départemental d'Analyses Agricoles, 39800 Poligny.

Baraud J., Emassieux S., Maurice A., 1970. Biosynthèse des acides gras à chaîne impaire ou ramifiée chez la levure Schizosaccharomyces liquefaciens. Rev. Fr. Corps Gras, 17, 155-163.

Berdague J.L., 1986. Méthode d'extraction rapide des acides gras volatils libres des fromages. Lait, 66 (3), 233-246.

Снамва J.F., 1984. Manifestation de la fermentation butyrique dans l'Emmental. Tech. Lait., (984), 9-14.

Choisy C., Desmazeaud M.J., Gripon J.C., Lamberet G., Lenoir J., Tourneur C., 1984. Les phénomènes microbiologiques et enzymatiques de la biochimie de l'affinage. Dans : Le Fromage, A. Eck, ed. Apria-Lavoisier, Paris, 62-89.

CLARK W.M., 1912. U.S. Department of Agriculture, Bulletin 151.

Deiana P., Fatichenti F., Farris G.A., Mocquot G., Lodi R., Todesco R., Cecchi L., 1984. Metabolization of lactic and acetic acids in Pecorino Romano cheese made with a combined starter of lactic acid bacteria and yeast. Lait, 64, 380-394. 
Favre-Bonvin J., Arpin N., 1984. Une nouvelle méthode de dosage des acides gras volatils. Rev. Lait. Fr., (434), 38-40.

Grappin R., Berdague J.L., Dasen A., Jeunet R., 1987. Affinage et qualité du Gruyère de Comté. I : Protocole expérimental. Caractérisation des conditions d'affinage et des ateliers de fabrication. Lait, 67 (2), 219-235.

Institut Technique du Gruyère, 1980. Aptitude de l'Emmental affiné à la conservation. Influence de la température, profil fermentaire. Etude S/80/1/C.

Kuchroo C.N., Fox P.F., 1982. Soluble nitrogen in Cheddar cheese : comparison of extraction procedures. Milchwissenschaft, 37, 331-335.

Kurz G., Wallenfels K., 1974. Methods of enzymatic analysis. Bergmeyer HU. ed. Academic Press-Inc. New York and London, 3, 1180-1184 et 1279 à 1282.

Kuzdzal-Savoie S., Kuzdzal W., 1966. Contribution à l'étude de la lipolyse dans les fromages. Tech. Lait., (14), 17-20.

KuzdZal-Savoie S., Kuzdzal W., 1967. Les acides gras libres du fromage. Lait, 47, (461-462), 923.

Kuzdzal-Savoie S., Kuzdzal W., 1971. Dosage des acides gras libres volatils dans quelques types de fromages français. Techn. Lait, (717), 11-14.

Kuzdzal-Savoie S., KuzDzal W., 1974. L'analyse par chromatographie en phase gazeuse des acides gras volatils libres des fromages et l'appréciation organoleptique. Spectra 2000, 9, 6266.

Masson C., Decaen C., Rousseaux P., Bouty J.L., 1978. Variations géographique et saisonnière de la composition du lait destiné à la fabrication du Gruyère de Comté. Lait, 58, (575-576), 261-273.

Mayenobe D., Didienne R., Pradel G., 1983. Caractérisation des gonflements tardifs dans les fromages de Saint-Nectaire et certaines pâtes pressées. Lait, 63, 15-24.

Moceuot G., 1979. Reviews of the progress of dairy science : Swiss-type cheese. J. Dairy Res., $46,133-160$.

StefFen C., Blanc B., 1971. Methoden zur Bestimmung der Gesamtmilchsäure und der Lactatkonfiguration in Käse und Milch. Schweiz. Milchztg., 97, 1073-1078.

Turner K.W., Thomas T.D., 1980. Lactose fermentation in Cheddar cheese and the effect of salt. N.Z.J. Dairy Sci. Technol., 15, 265-276.

VAN Niel C.B., 1928. The propionic acid bacteria. J.W. BoIsSEVIAN and Co, Haarlem (Thesis : Delft).

Wood H.G., Werkman C.H., 1937. Assimilation of pyruvic acid by the propionic acid bacteria. Iowa State Coll. J. Sci., 11, 287-292. 